\title{
EXAMPLES IN THE THEORY OF SUFFICIENCY OF JETS
}

\author{
WOJCIECH KUCHARZ
}

\begin{abstract}
It is shown that for a given nonnegative integer $s$, there exist a positive integer $r(s)$ and an $r(s)$-jet $v_{s}$ with source at $O \in \mathbf{R}^{3}$ which is not $V$-sufficient in the class of $C^{r(s)+s}$-realizations and is $C^{0}$-sufficient in the class of $C^{r(s)+s+1}$-realizations. In the complex case, a jet with source at $O \in \mathbf{C}^{2}$ which is $V$-sufficient but not $C^{0}$-sufficient in the class of holomorphic realizations is constructed.
\end{abstract}

1. Introduction. Denote by $E^{r}(n, 1)$ the set of all germs of $C^{r}$ functions $\left(\mathbf{R}^{n}, 0\right) \rightarrow(\mathbf{R}, 0)$. Two germs $f, g \in E^{r}(n, 1)$ are said to be $V$-(resp. $C^{0}$-) equivalent if the set-germs $f^{-1}(0), g^{-1}(0)$ are homeomorphic (resp. $g=f \circ \sigma$ for some local homeomorphism $\left.\sigma:\left(\mathbf{R}^{n}, 0\right) \rightarrow\left(\mathbf{R}^{n}, 0\right)\right)$. We shall not distinguish between germs and representative functions. Let $J^{r}(n, 1)$ be the set of $r$-jets of all germs belonging to $E^{r}(n, 1)$. Given a jet $w \in J^{r}(n, 1)$, a germ $f \in E^{r+s}(n, 1), s \geq 0$, whose $r$-jet $j^{r} f$ is equal to $w$, is said to be a $C^{r+s_{-}}$realization of $w$. The jet $w$ is called $V$-(resp. $\left.C^{0}-\right)$ sufficient in $E^{r+s}(n, 1)$ if any two of its $C^{r+s}$-realizations are $V$-(resp. $C^{0_{-}}$) equivalent. We shall identify $r$-jets with their polynomial representatives of degree not exceeding $r$.

Problems concerning sufficiency of jets have been studied by many authors. A number of criteria and characterizations have been found in the case $s=0,1$. Recall the following result.

ThEOREM A $[\mathbf{4}, \mathbf{9}, \mathbf{1 1}]$. For any $r$-jet $w \in J^{r}(n, 1)$, the following conditions are equivalent:

(a) $w$ is $V$-sufficient in $E^{r}(n, 1)\left(\right.$ resp. $\left.E^{r+1}(n, 1)\right)$;

(b) $w$ is $C^{0}$-sufficient in $E^{r}(n, 1)\left(\right.$ resp. $\left.E^{r+1}(n, 1)\right)$;

(c) there exist $c, \varepsilon, \delta>0$ such that

$$
|\nabla w(x)| \geq c|x|^{r-1} \quad\left(\text { resp. }|\nabla w(x)| \geq c|x|^{r-\delta}\right) \quad \text { for }|x|<\varepsilon .
$$

It is also known that sufficiency in $E^{r+1}(n, 1)$ does not imply sufficiency in $E^{r}(n, 1)[4]$.

A natural problem, that of finding reasonable conditions characterizing $r$-jets sufficient in $E^{r+s}(n, 1)$ for $s \geq 2$, seems to be complicated. The following example supports this opinion.

EXAMPLE 1.1. Let $s$ be a nonnegative integer and let $h_{s}:\left(\mathbf{R}^{3}, 0\right) \rightarrow(\mathbf{R}, 0)$ be the germ at 0 of the polynomial function $x_{1}^{3}-3 x_{1} x_{2}^{2(s+2)+1}+x_{3}^{3}$. Put $r(s)=2(s+3)+1$. Then the $(r(s)-1)$-jet $u_{s}$ and $(r(s)+s)$-jet $w_{s}$ of $h_{s}$ have the following properties:

(a) $u_{s}$ is $C^{0}$-sufficient in $E^{r(s)+s+1}(3,1)$;

(b) $w_{s}$ is not $V$-sufficient in $E^{r(s)+s}(3,1)$.

In particular, the $r(s)$-jet $v_{s}$ of $h_{s}$ is not $V$-sufficient in $E^{r(s)+s}(3,1)$ (in fact there

Received by the editors February 25, 1985.

1980 Mathematics Subject Classification. Primary 57R45, 58E15.

(C)1986 American Mathematical Society $0002-9939 / 86 \$ 1.00+\$ .25$ per page 
exists a sequence $\left\{f_{i}\right\}, i=1,2, \ldots$, of $C^{r(s)+s}$-realizations of $v_{s}$ with $f_{i}$ and $f_{j}$ not $V$-equivalent for $i \neq j)$, but is $C^{0}$-sufficient in $E^{r(s)+s+1}(3,1)$. Moreover, $r(s)+s-1<L\left(v_{s}\right)<r(s)+s$, where given a function $f$ in a neighborhood of $0 \in \mathbf{R}^{n}, L(f)$ denotes the smallest positive real number $\alpha$ such that $|\nabla f(x)| \geq c|x|^{\alpha}$ in a neighborhood of 0 for some $c>0$.

We shall prove all these properties in $\S 2$. The example shows that a result analogous to Theorem A cannot be true for $s \geq 2$. In particular, conjecture 1 of [2], that an $r$-jet is $V$-(resp. $\left.C^{0}-\right)$ sufficient in $E^{r+s}(n, 1), s \geq 2$, if and only if it is $V$-(resp. $C^{0}$-) sufficient in $E^{r+1}(n, 1)$, is false.

We shall also study sufficiency of complex jets. Denote by $H(n, 1)$ and $J_{\mathbf{C}}^{r}(n, 1)$ the space of all germs of holomorphic functions $\left(\mathbf{C}^{n}, 0\right) \rightarrow(\mathbf{C}, 0)$ and the space of all complex $r$-jets of such germs, respectively. The notions of $V$ - and $C^{0}$-sufficiency of complex $r$-jets in $H(n, 1)$ can be defined in the obvious way. In the complex case, it is convenient to introduce a new concept which occupies an intermediate position between $V$ - and $C^{0}$-sufficiency. An $r$-jet $w \in J_{\mathbf{C}}^{r}(n, 1)$ is called $S V$-sufficient (strongly $V$-sufficient) in $H(n, 1)$ if for any two holomorphic realizations $f$ and $g$ of $w$ there exists a local homeomorphism $\left(\mathbf{C}^{n}, 0\right) \rightarrow\left(\mathbf{C}^{n}, 0\right)$ transforming $f^{-1}(0)$ onto $g^{-1}(0)$. Finally, given a germ $f \in H(n, 1)$, we denote by $\mu(f)$ its Milnor number

$$
\mu(f)=\operatorname{dim}_{\mathbf{C}} H(n) / \Delta(f),
$$

where $H(n)$ is the $\mathbf{C}$-algebra of all germs of holomorphic functions $\left(\mathbf{C}^{n}, 0\right) \rightarrow \mathbf{C}$ and $\Delta(f)$ is the ideal of $H(n)$ generated by the first partial derivatives of $f$.

The following characterization is known.

THEOREM B [2]. Given a complex $r$-jet $w \in J_{\mathbf{C}}^{r}(n, 1)$, the following conditions are equivalent:

(a) $w$ is $S V$-sufficient in $H(n, 1)$;

(b) $w$ is $C^{0}$-sufficient in $H(n, 1)$;

(c) there exist $c, \varepsilon, \delta>0$ such that $|\nabla w(z)| \geq c|z|^{r-\delta}$ for $|z|<\varepsilon$;

(d) for every holomorphic realization $f$ of $w, \mu(f)=\mu(w)$.

One might think that condition (a) in Theorem B could be replaced by a weaker one, that $w$ is merely $V$-sufficient in $H(n, 1)$. However, we have the following example which will be discussed in detail in $\S 2$.

EXAMPLE 1.2. The jet $w=z_{1}^{4}-4 z_{1} z_{2}^{8} \in J_{\mathbf{C}}^{9}(2,1)$ is $V$-sufficient but is not $S V$-sufficient in $H(2,1)$.

2. Proofs. A crucial part in our proofs depends on computing the Milnor number of certain germs.

A complex polynomial $\psi\left(z_{1}, \ldots, z_{n}\right)$ is said to be quasi-homogeneous of type $\left(\alpha_{1}, \ldots, \alpha_{n}\right)$, where $\alpha_{j}$ is a rational number, $0<\alpha_{j} \leq 1 / 2$, if it is a C-linear combination of monomials $z_{1}^{k_{1}} \cdots z_{n}^{k_{n}}$ with $\alpha_{1} k_{1}+\cdots+\alpha_{n} k_{n}=1$. We shall use the following result of $\mathrm{V}$. Arnol'd.

Theorem C [1, Theorem 3.1, Corollary 4.8]. Suppose that a germ $\varphi \in H(n, 1)$ is in the form

$$
\varphi=\varphi_{0}+\varphi_{1}
$$


where $\varphi_{0}$ is a homogeneous polynomial of type $\left(\alpha_{1}, \ldots, \alpha_{n}\right)$ with an isolated critical point at $0 \in \mathbf{C}^{n}$ and for any monomial $c z_{1}^{j_{1}} \cdots z_{n}^{j_{n}}, c \neq 0$, in the Taylor expansion of $\varphi_{1}, \alpha_{1} j_{1}+\cdots+\alpha_{n} j_{n}>1$. Then

$$
\mu(\varphi)=\mu\left(\varphi_{0}\right)=\left(1 / \alpha_{1}-1\right) \cdots\left(1 / \alpha_{n}-1\right) .
$$

Consider Example 1.1. (a) Denote by $\psi$ the complexification of $h_{s}$. Let $\varphi \in$ $H(3,1)$ be a germ of a holomorphic function with the same $(r(s)-1)$-jet as $\psi$. Then $\varphi$ can be written as

$$
\varphi=\psi+a_{1} z_{2}^{r(s)}+\cdots+a_{s+1} z_{2}^{r(s)+s}+\gamma,
$$

where $a_{j} \in \mathbf{C}, \gamma \in H(3,1)$, the $(r(s)-1)$-jet of $\gamma$ vanishes, and the Taylor expansion of $\gamma$ does not contain terms of the form $b z_{2}^{k}, b \neq 0, k \leq r(s)+s$.

Two cases can occur.

(i) If $a_{1}=\cdots=a_{s+1}=0$, then $\varphi=\varphi_{0}+\varphi_{1}$ is in the form (2.1), where $\varphi_{0}=\psi$ is a quasi-homogeneous polynomial of type $(1 / 3,2 /(6 s+15), 1 / 3), \varphi_{1}=\gamma$ and $\mu(\varphi)=\mu\left(\varphi_{0}\right)=2(6 s+13)$.

(ii) If $a_{1}=\cdots=a_{k}=0$ and $a_{k+1} \neq 0$ for some $k, 0 \leq k \leq s$, then $\varphi=\varphi_{0}+\varphi_{1}$ is in the form (2.1), where $\varphi_{0}=z_{1}^{3}+a_{k+1} z_{2}^{r(s)+k}+z_{3}^{3}$ is a quasi-homogeneous polynomial of type $(1 / 3,1 /(r(s)+k), 1 / 3), \varphi_{1}=a_{k+2} z_{2}^{r(s)+k+1}+\cdots+a_{s+1} z_{2}^{r(s)+s}-$ $3 z_{1} z_{2}^{r(s)}+\gamma$ and $\mu(\varphi)=\mu\left(\varphi_{0}\right)=4(2(s+3)+k)$.

Note that in both cases $\mu(\varphi)=\mu\left(j^{r(s)+s} \varphi\right)$. Hence, by Theorem B, there exist $c, \varepsilon, \delta>0$ such that

$$
|\nabla \varphi(z)| \geq c|z|^{r(s)+s-\delta} \text { for }|z|<\varepsilon .
$$

Now let $f$ be a $C^{r(s)+s+1}$-realization of $u_{s}$. We shall show that $f$ is a topological projection; i.e., $(f \circ \sigma)\left(x_{1}, x_{2}, x_{3}\right)=x_{1}$ for some local homeomorphism $\sigma:\left(\mathbf{R}^{3}, 0\right) \rightarrow$ $(\mathbf{R}, 0)$. It follows from Theorem $\mathrm{A}$ and $(2.2)$ that the $(r(s)+s)$-jet of $f$ is $C^{0}$ sufficient in $E^{r(s)+s+1}(3,1)$. Thus we may assume that $f$ is a polynomial function. The complexification $\varphi$ of $f$ can be written as $\varphi=\varphi_{0}+\varphi_{1}$, where $\varphi_{0}$ and $\varphi_{1}$ are as in (i) or (ii). Consider the family of germs $G_{t}=\varphi_{0}+t \varphi_{1}, t \in[0,1]$. By Theorem $\mathrm{C}, \mu\left(G_{t}\right)=\mu\left(\varphi_{0}\right)$ and hence there exists a neighborhood $U$ of 0 in $\mathbf{C}^{3}$ such that for each $t$ in $[0,1], 0$ is the only critical point of $G_{t}$ in $U$. Observe that the restriction $F_{t}$ of $G_{t}$ to $U \cap \mathbf{R}^{3}$ is a real analytic function and $0 \in \mathbf{R}^{3}$ is its unique critical point. By King's theorem [7, Theorem 1, Corollary 1] the family of germs $F_{t}:\left(\mathbf{R}^{3}, 0\right) \rightarrow(\mathbf{R}, 0)$ is topologically trivial; i.e., $F_{t} \circ \sigma_{t}=F_{0}$ for some continuous family of local homeomorphisms $\sigma_{t}:\left(\mathbf{R}^{3}, 0\right) \rightarrow\left(\mathbf{R}^{3}, 0\right)$. In particular, $f \circ \sigma_{1}=F_{0}$. Thus $f \circ \sigma_{1}=h_{s}$ if $\varphi$ is in the form (i), and $f \circ \sigma_{1}=x_{1}^{3}+a_{k+1} x_{2}^{r(s)+k}+x_{3}^{3}$ if $\varphi$ is in the form (ii). In both cases $f \circ \sigma_{1} \circ \tau$ is a germ of a real analytic function, where $\tau:\left(\mathbf{R}^{3}, 0\right) \rightarrow\left(\mathbf{R}^{3}, 0\right)$ is the local homeomorphism defined by $\tau\left(x_{1}, x_{2}, x_{3}\right)=$ $\left(x_{1}, x_{2}, x_{3}^{1 / 3}\right)$. Clearly, $0 \in \mathbf{R}^{3}$ is a regular point of $f \circ \sigma_{1} \circ \tau$, and hence $f$ is a topological projection.

(b) The germ $f=h_{s}+2 x_{2}^{r(s)+s+1 / 2}$ is a $C^{r(s)+s}$-realization of $w_{s}$ and

$$
(\nabla f)\left(x_{1}, x_{2}, x_{3}\right)=0 \quad \text { for } x_{1}=x_{2}^{s+2+1 / 2}, x_{3}=0 .
$$

Hence, by Theorem $\mathrm{A}$, the jet $w_{s}$ is not $V$-sufficient in $E^{r(s)+s}(3,1)$. 
From (a) and (b), it is clear that the jet $v_{s}$ is not $V$-sufficient in $E^{r(s)+s}(3,1)$ and is $C^{0}$-sufficient in $E^{r(s)+s+1}(3,1)$. Theorem $\mathrm{A}$ implies that $r(s)+s-1<L\left(v_{s}\right)<$ $r(s)+s$. By a theorem of Bochnak and Kuo [3] there exists a sequence $\left\{f_{i}\right\}$, $i=1,2, \ldots$, of $C^{r(s)+s}$-realizations of $w_{s}$ such that $f_{i}$ and $f_{j}$ are not $V$-equivalent for $i \neq j$. Of course, each $f_{i}$ is also a $C^{r(s)+s}$-realization of $v_{s}$.

Consider Example 1.2. Let $\varphi$ be a holomorphic realization of $w$. Using essentially the same techniques as in the proof of condition (a) of Example 1.1, one can find a local homeomorphism $\sigma:\left(\mathbf{C}^{2}, 0\right) \rightarrow\left(\mathbf{C}^{2}, 0\right)$ such that $\varphi \circ \sigma=\psi_{1}$, where $\psi_{1}=w$ or $\varphi \circ \sigma=\psi_{2}$, where $\psi_{2}=z_{1}^{4}-z_{2}^{10}$. Note that the germs at 0 of $\psi_{1}^{-1}(0)$ and $\psi_{2}^{-1}(0)$ are homeomorphic to the germ at 0 of the set $\left\{\left(z_{1}, z_{2}\right) \in \mathbf{C}^{2} \mid z_{1} z_{2}=0\right\}$. This means that $w$ is $V$-sufficient in $H(2,1)$. Since $\mu(w)=29$ and $\mu\left(w+z_{2}^{10}\right)=27, w$ is not $S V$-sufficient.

\section{REFERENCES}

1. V. I. Arnol'd, Normal forms in neighborhoods of degenerate critical points, Russian Math. Surveys 29 (1974), 10-50.

2. J. Bochnak and W. Kucharz, Sur les germs d'applications différentiables à singularités isolées, Trans. Amer. Math. Soc. 252 (1979), 115-131.

3. J. Bochnak and T. C. Kuo, Different realizations of nonsufficient jet, Indag. Math. 34 (1972), 24-31.

4. J. Bochnak and S. Eojasiewicz, A converse of the Kuiper-Kuo theorem, Lecture Notes in Math., vol. 192, Springer-Verlag, Berlin and New York, 1971, pp. 254-261.

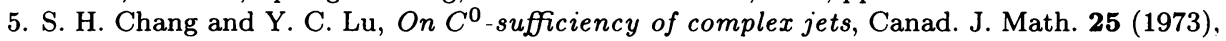
874-880.

6. T. Fukuda, Types topologiques des polynômes, Inst. Hautes Études Sci. Publ. Math. 46 (1976), 87-106.

7. H. King, Topological type in families of germs, Invent. Math. 62 (1980), 1-13.

8. S. Koike and W. Kucharz, Sur les réalisations de jets non-suffisants, C. R. Acad. Sci. Paris Sér. I Math. 288 (1979).

9. T. C. Kuo, On $C^{0}$-sufficiency of jets of potential functions, Topology 8 (1969), 167-171.

10. __ Characterizations of $V$-sufficiency of jets, Topology 11 (1972), 115-131.

11. N. Kuiper, $C^{1}$-equivalence of functions near isolated critical points, Sympos. Infinite Dimensional Topology (Baton Rouge, 1967), Princeton Univ. Press, Princeton, N. J.; Univ. of Tokyo Press, Tokyo, 1972, pp. 199-218.

12. D. T. Lê and C. P. Ramanujam, The invariance of Milnor's number implies the invariance of topological type, Amer. J. Math. 98 (1976), 67-78.

13. J. Milnor, Singular points of complex hypersurfaces, Ann of Math. Stud., vol. 61, Princeton Univ. Press, Princeton, N.J., 1968.

Department of Mathematics and Statistics, University of NeW Mexico, Albuquerque, NeW MeXico 87131 